\title{
Nationales Forschungsprogramm verdeutlicht Herausforderungen
}

\section{Jean Martin}

Dr. med., Mitglied der Redaktion

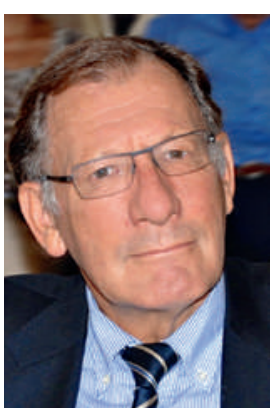

Das Nationale Forschungsprogramm «Lebensende» (NFP 67) wurde im Jahr 2011 auf fünf Jahre und mit einem Finanzrahmen von 15 Millionen aufgelegt. Ausgewählt wurden 33 Forschungsprojekte. Im Jahr 2016 wurden fünf Dialogveranstaltungen organisiert, im Rahmen deren die Ergebnisse diskutiert werden. Die erste Veranstaltung "Sterben zu Hause und im Heim» fand am 19. Februar in Bern statt. Dabei wurde vor allem die Rolle der pflegenden Angehörigen thematisiert, die vermehrt vor der Aufgabe stehen, ihre alten Eltern zu betreuen, eine Aufgabe, die zunehmend an Bedeutung gewinnt. Eine in Freiburg und im Wallis angestrengte Studie unter der Leitung von B. Sottas hat die in diesem Zusammenhang kritischen Zusammenhänge untersucht. Es zeigte sich, dass die Dauer der externen Pflege mit etwas über einer Stunde pro Tag anzusetzen ist, während pflegende Angehörige permanent verfügbar sein müssen. Dabei haben Letztere oft Mühe, den Überblick zu behalten, denn pro Patient können bis zu 32 Akteure auftreten, was ein hohes Mass an Koordination voraussetzt. Die Studie kommt u.a. zu dem Schluss, dass die Angehörigen (mehrheitlich pflegen die Frauen) häufig physisch und psychisch erschöpft sind und das Gefühl haben, allein dazustehen. Sie können sich nur schwer auf das absehbare Ableben des Patienten vorbereiten, haben Angst, Dinge nicht richtig oder in ausreichendem Masse zu tun, und befürchten, die Kontrolle über die Situation und ihr eigenes Leben zu verlieren.

Eine in Lausanne unter der Leitung von M.-A. Berthod durchgeführte Studie befasste sich damit, wie - und obes den Angehörigen gelingt, Berufsleben und Pflegeleistung unter einen Hut zu bringen. Die erfassten persönlichen Geschichten zeigen auf, wie sich die Betreffenden arrangieren. Dabei überrascht es nicht, dass sich die getroffenen Massnahmen mehr oder weniger einfach - oder schwierig - gestalten, je nach Unternehmenskultur und gutem Willen der Vorgesetzten. Die Autoren unterstreichen, dass es besonders wichtig ist, möglichst auf allen Ebenen besser strukturierte Mittel und Wege bereitzustellen, um die Verfügbarkeit der pflegenden Angehörigen zu erleichtern. Dazu zählen u.a. der Schutz des Arbeitsplatzes für jene, die ihre Arbeitszeit reduzieren, aber auch organisatorische und materielle Unterstützung. In diesem Zusammenhang wird im Herbst 2016 die nationale Plattform «Work and Care» auf den Weg gebracht. Ehrenamtliche können dort eingesetzt werden, unter der Voraussetzung, dass deren Einbindung zuverlässig und entsprechend koordiniert ist.

Die von A.-V. Dürst, S. Monod et coll. (Lausanne) vorgelegte Forschungsarbeit befasste sich mit dem bei alten Patienten vorhandenen Wunsch zu sterben: Bei Patienten in der REHA lag dieser Wunsch bei 12,9\% und im Heim bei $21 \%$. In diesem Zusammenhang wurde die Frage nach der Sterbehilfe aufgeworfen. Die Zahlen in der Schweiz machen deutlich, dass Sterbehilfe häufiger bei den über 65-Jährigen in Anspruch genommen wurde (bei den über 75-Jährigen liegt die Zahl nahe der nichtassistierter Suizide). Die Autoren der Studie haben interessanterweise herausgefunden, dass sich der Wunsch zu sterben bei Menschen mit moderatem oder auch erhöhtem kognitivem Defizit adäquat bemessen lässt [1]. Drei Experten haben die vorgelegten Resultate kommentiert, darunter die Leiterin der Abteilung für Strategie und Grundsatzfragen des BAG. Die Herausforderungen sind komplexer Natur, so viel lässt sich zumindest sagen. Zum einen gibt es eine Vielzahl von Akteuren. Diese gilt es zu reduzieren, ohne dabei die Pflegequalität zu beeinträchtigen. Die Rolle der pflegenden Angehörigen verlangt zwingend nach mehr Anerkennung. Ausserdem ist verstärkt darauf zu achten, wie sie unterstützt werden können: Harmonisierung der Pflege mit dem Berufsleben, Entlastung durch Dritte, Informationsvermittlung und entsprechende Schulungen. Im praktischen Alltag obliegen die Aufgaben verschiedenen privaten und öffentlichen Leistungsanbietern. Im öffentlichen Bereich variieren die Zuständigkeiten je nach Kanton und fallen dabei den Gemeinden, Bezirken oder dem Staat zu. In der Schweiz ist man an diese - oft politisch tief verwurzelte - Vielfalt gewöhnt. Dabei ist jedoch dafür Sorge zu tragen, dass unsere alten und abhängigen Mitbürger überall und gleichermassen Zugang zu qualitativ und quantitativ angemessenen Leistungen haben. Die Herausforderungen, vor die wir durch den "grauen Tsunami» gestellt werden, sind gewaltig. Daher ist es gut, dass das NFP 67 jene Bereiche aufzeigt, die der dringenden Beachtung bedürfen. 